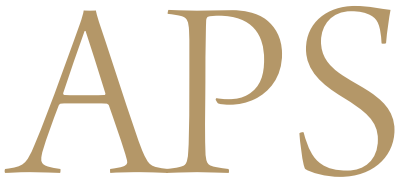

Archives of Plastic Surgery

\title{
Fingertip Reconstruction Using Free Toe Tissue Transfer Without Venous Anastomosis
}

\author{
Won Young Yoon, Byung Il Lee \\ Department of Plastic and Reconstructive Surgery, Korea University College of Medicine, Seoul, Korea
}

Background This study was designed to introduce the feasibility of toe tissue transfer without venous outflow for fingertip reconstruction.

Methods Five cases of fingertip defects were treated successfully with this method. Four cases were traumatic fingertip defects, and one case was a hook-nail deformity. The lateral pulp of a great toe or medioinferior portion of a second toe was used as the donor site. An arterial pedicle was dissected only within the digit and anastomosis was performed within $2 \mathrm{~cm}$ around the defect margin. The digital nerve was repaired simultaneously. No additional dissection of the dorsal or volar pulp vein was performed in either the donor or recipient sites. Other surgical procedures were performed following conventional techniques. Postoperative venous congestion was monitored with pulp temperature, color, and degree of tissue oxygen saturation. Venous congestion was decompressed with a needle-puncture method intermittently, but did not require continuous external bleeding for salvage.

Results Venous congestion was observed in all the flaps, but improved within 3 or 4 days postoperatively. The flap size was from $1.5 \times 1.5 \mathrm{~cm}^{2}$ to $2.0 \times 3.0 \mathrm{~cm}^{2}$. The mean surgical time was 2 hours and 20 minutes. A needle puncture was carried out every 2 hours during the first postoperative day, and then every 4 hours thereafter. The amount of blood loss during each puncture procedure was less than $0.2 \mathrm{~mL}$. In the long-term follow-up, no flap atrophy was observed.

Conclusions When used properly, the free toe tissue transfer without venous anastomosis method can be a treatment option for small defects on the fingertip area.

\author{
Correspondence: Byung II Lee \\ Department of Plastic and \\ Reconstructive Surgery, Korea \\ University College of Medicine, \\ 73 Inchon-ro, Seongbuk-gu, Seoul \\ 136-705, Korea \\ Tel: +82-2-920-5698 \\ Fax: +82-2-922-7437 \\ E-mail: guro@korea.ac.kr
}

Keywords Finger injuries / Free tissue flaps / Hyperemia

This article was presented the 11th Triennial Congress of the International Federation of Societies for Surgery of the Hand on November 4, 2010 in Seoul, Korea.

No potential conflict of interest relevant to this article was reported.

\section{INTRODUCTION}

Fingertips are highly susceptible to trauma, but they are also an essential part of the hand for both functional and esthetic reasons. Since the first successful finger replantation by Komatsu and Tamai [1], there has been much progress in microsurgical fingertip replantation and reconstruction distal to the distal interphalangeal joint [2]. However, in distal digital microsurgery, the main technical difficulty is venous repair [3]. This is due to its smaller diameter and the flaccidity of the vessel wall compared to the arteries $[4,5]$. There also is a risk for postoperative occlusion of the anastomosed vein. Especially when the dorsal veins are severely damaged, and thus, not good candidates for revascularization, the problems could be even more complicated. Furthermore, sometimes surgeons are not inclined to use the dorsal vein as an anastomosed pedicle because the 
injured part is localized in the volar aspect. Successful fingertip replantation by repairing only the digital artery without venous anastomosis has been reported on occasionally in the literature $[6,7]$. To maintain outflow from artery-only replants until venous outflow is restored naturally, multiple techniques have been described including periodic puncturing [8]. Given the safety and efficacy of artery-only anastomosis in distal digital replantation, we adopted this approach for reconstruction of the fingertip area with free toe tissue transfer.

Therefore, this study was carried out to evaluate the feasibility of free toe tissue transfer without venous repair in fingertip reconstruction.

\section{METHODS}

A total of 5 patients were included in the study, consisting of 2 males and 3 females (Table 1). The cases included one thumb, two index fingers, and two middle fingers. All of the patients underwent primary reconstruction after amputation except the case of hook-nail deformity on the index finger. All of the operations were performed under general anesthesia and by the same surgical team. Donor tissues were harvested with the traditional free toe tissue transfer methods. In 2 cases, donor tissue was harvested from the lateral side of the great toes; in the rest, it was harvested from the mediovolar side of the second toes.

The proximal amputated portion was prepared under a surgical microscope, and sufficient arterial bleeding was confirmed before arterial anastomosis. Additional dissection was not necessary at the proximal portion for reconstruction of the amputated finger, but for the hook-nail deformity it was partially performed to release scar contracture simultaneously. With the use of a finger tourniquet, only proper plantar digital arteries were anastomosed using 10-0 nylon sutures. Arterial anastomoses were performed within the wound or within $2 \mathrm{~cm}$ proximal to the defect. The digital nerves were repaired to improve sensation and decrease symptoms of neuroma.

Venorrhaphy was not performed in any of the cases; hence no additional incision or dissection for volar vein anastomosis was

\begin{tabular}{|c|c|c|c|c|c|c|}
\hline Case & $\begin{array}{l}\text { Sex/ } \\
\text { Age }\end{array}$ & Site & $\begin{array}{l}\text { Mechanism } \\
\text { of injury }\end{array}$ & $\begin{array}{l}\text { Donor } \\
\text { site }\end{array}$ & $\begin{array}{c}\text { Flap size } \\
\left(\mathrm{cm}^{2}\right)\end{array}$ & VAS \\
\hline 1 & $M / 35$ & Index & Pulp amputation & Second toe & $2.0 \times 2.0$ & 9.5 \\
\hline 2 & $\mathrm{~F} / 45$ & Thumb & Crushing injury & Great toe & $2.0 \times 2.0$ & 9.3 \\
\hline 3 & $F / 21$ & Index & Hooknail deformity & Second toe & $1.5 \times 3.0$ & 8.5 \\
\hline 4 & $M / 53$ & Middle & Crushing injury & Second toe & $1.5 \times 1.5$ & 8.8 \\
\hline 5 & $\mathrm{M} / 34$ & Middle & Degloving amputation & Great toe & $2.0 \times 3.0$ & 9.0 \\
\hline
\end{tabular}

needed in the harvested flaps or recipient sites. After surgery, the patients were placed in a warm room with a heat lamp, and their hands were elevated above the level of their hearts. The patients were administered $500 \mathrm{~mL}$ of low-molecular weight dextran intravenously for 5 consecutive days, but heparin was not used.

Temperature, color, and percutaneous oxygen saturation was monitored and compared with the corresponding contralateral digit to evaluate postoperative venous congestion. Change in flap color or significant flap fullness was regarded as a sign of congestion, and drainage was performed using periodic puncture of the fingertip. Within 24 hours postoperatively, periodic pin-pricking was carried out every 2 hours. After 24 hours, 4-hour intervals were sufficient for venous drainage. Pin-pricking had been continued for 5 days in the case-4-patient, but it was sufficient to relieve venous congestion for 3 days in the other cases.

Moreover, patency of the anastomosed artery was established by monitoring capillary refilling and the Doppler sound. Every patient was informed about the importance of smoking cessation after surgery to prevent poor surgical outcomes.

The functional and cosmetic outcomes were assessed on postoperative follow-ups. The patients' satisfaction was also evaluated using a visual analogue scale, with a score of 0 being the worst and 10 being the best.

\section{RESULTS}

Flap sizes ranged from $1.5 \times 1.5 \mathrm{~cm}^{2}$ to $2.0 \times 3.0 \mathrm{~cm}^{2}$, and the mean operation time was 2 hours and 20 minutes. Arterial anastomosis was successful in all 5 patients, and vein grafting for arterial repair was not necessary in any of the cases. None of the cases showed arterial insufficiency or flap loss.

In all the cases, venous congestion was noted in postoperative monitoring, but the patients recovered in 3 to 5 days without secondary procedures except periodic pin-pricking. The amount of blood loss was less than $0.2 \mathrm{~mL}$ during each puncture procedure; therefore, blood transfusion was not required in any patients. The mean hospital stay was approximately 10 days.

The mean postoperative follow-up period was 7 months, and significant flap atrophy was not observed. The mean score of patient satisfaction was $9.02 \pm 0.40$ measured using a visual analogue scale.

Neither pain nor significant sensory loss was observed in any of the cases.

\section{Case 1}

A 35-year-old male amputated his right index fingertip. A primarily composite graft was performed, but it failed with necrotic change. Reconstruction was performed with the mediovolar 


\section{Fig. 1. Index fingertip reconstruction using second toe tissue}

Right index fingertip was reconstructed with second toe tissue in the case 1 patient. (A) Preoperative state. Initially a composite graft was performed but failed. (B) A $2.0 \times 2.0 \mathrm{~cm}^{2}$ sized flap was designed on the mediovolar aspect of the left second toe. (C) A toe flap, based only on the digital artery and nerve, was elevated. (D) The fingertip was well reconstructed with adequate volume and shape.
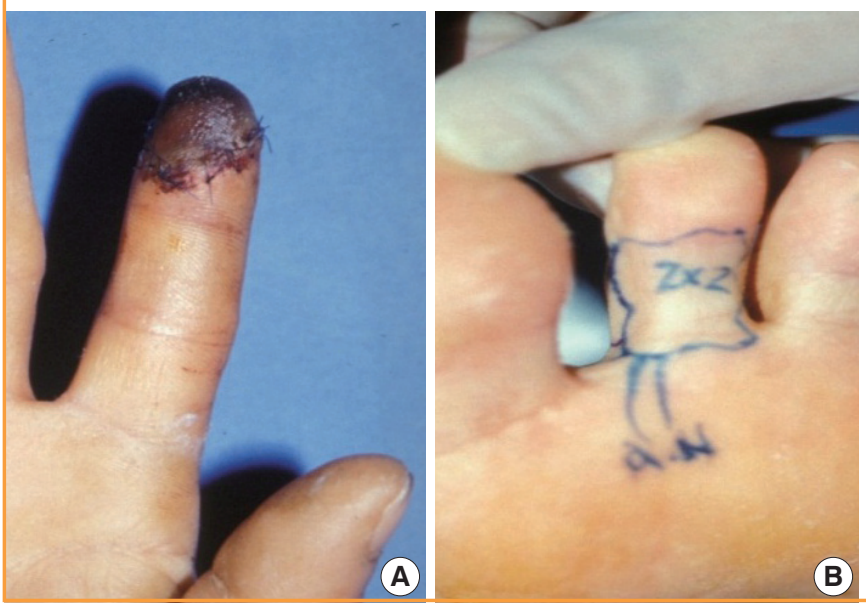

aspect of the left second toe and the tissue survived (Fig. 1).

\section{Case 2}

A 45-year-old female had a crushing amputation on her left thumb tip. The amputated tip was well reconstructed using left great toe tissue transfer (Fig. 2).

\section{Case 3}

A 21-year-old female had a hook-nail deformity on her right index finger (Fig. 3). Reconstruction using the mediovolar aspect of the left second toe was performed, and the hook-nail deformity was corrected.

\section{DISCUSSION}

Fingertip injury is one of the most common hand injuries, and numerous techniques have been attempted to treat it. Healing by primary closure and revision amputation can be the most straightforward method, but they can cause hook-nail deformity or neuroma formation at the stump. Skin grafts are another option, but only for defects without bone or tendon exposure [9] Various local flaps have been developed to reconstruct fingertips including V-Y flaps, cross-finger flaps, thenar flaps [10], and island flaps [11]. However, postoperative scar contracture or flexion deformity remained as sequelae, and sometimes those flaps are not possible for treating a tip defect such as a transversely amputated stump or pure pulp defect extended to the middle phalanx. Because advances in microsurgery allow options for microsurgical restoration of distal digits, free toe tissue transfer has become the superior choice over conventional techniques
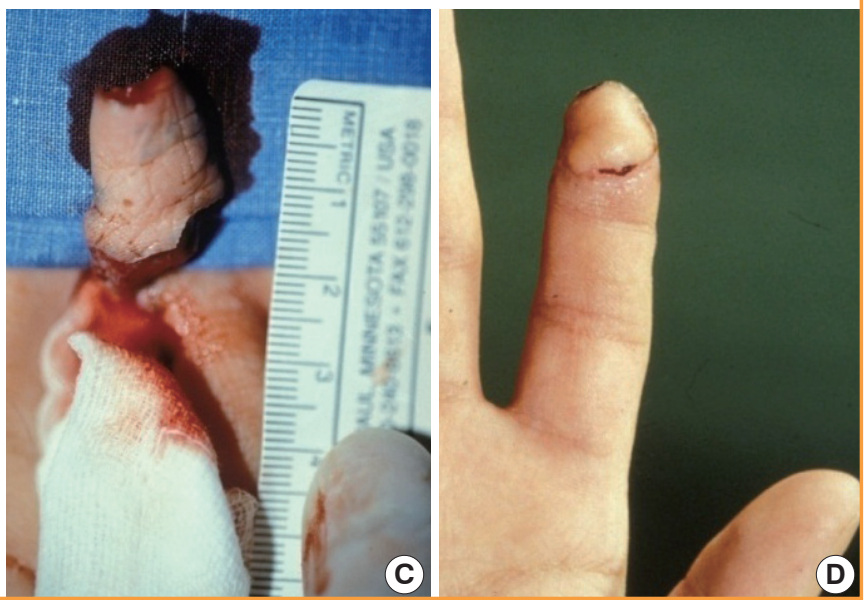

Fig. 2. Thumb tip reconstruction using great toe tissue

The left thumb tip was reconstructed with great toe tissue in the case 2 patient. (A) The distal half of the distal phalanx was amputated in the left thumb preoperatively. (B) The amputated tip was well reconstructed with sufficient padding and esthetic shape postoperatively.
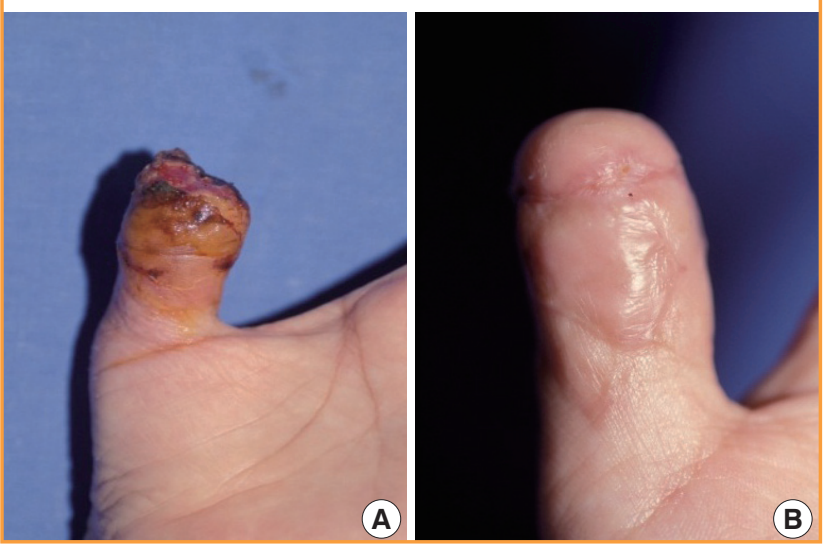

in esthetics and function [12].

Adequate venous outflow is one of the most important factors for successful free toe tissue transfer including distal digital reconstruction [3]. Of course it is the safest option if at least one successfully anastomosed vein is present. However, previous studies have provided convincing evidence that amputated fingertips can be salvaged successfully using only arterial anastomosis with simple surgical or nonsurgical methods of venous drainage [13-15]. We adopted this method for free toe tissue transfer for fingertip reconstruction. All 5 of our cases of free toe tissue transfer of artery-only anastomosis survived without significant complications. Periodic pin-pricking did not require blood transfusion or prolonged hospitalization. We believe 


\section{Fig. 3. Hooknail deformity correction using second toe tissue}

A hook-nail deformity was reconstructed well with second toe tissue transfer in the case 3 patient. (A, B) Preoperative view. Significant hook-nail deformity was noted. (C) Immediate postoperative view of a reconstructed fingertip. (D-F) Postoperative 6 months view. A hook-nail deformity was corrected, and the patient was satisfied with the results.
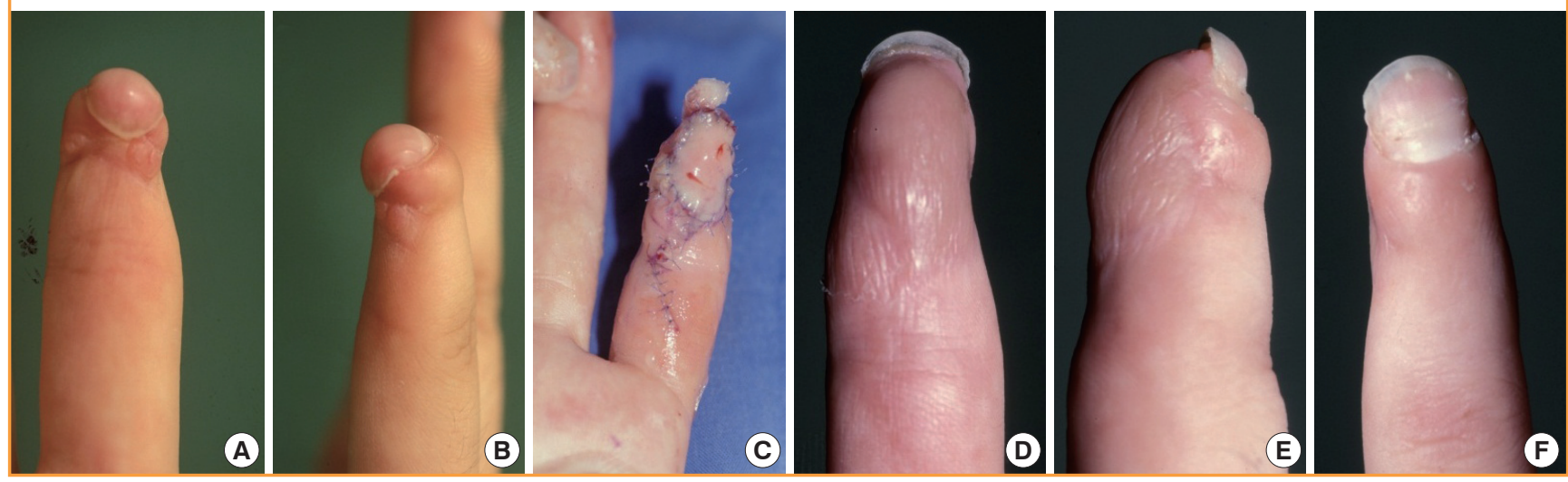

that this consistent result comes from the wide contact surface between the donor flap and recipient site compared to the flap volume. A wide contact surface enhances early revascularization of a replanted flap, and for that reason total flap survival was achieved.

In some ways our procedure could be considered a form of free composite graft. However, the donor tissue in a composite graft is totally devitalized because there is no blood flow initially. This is the biggest and the most important difference between a composite graft and our procedure. Although survival of a free tissue transfer is critically dependent on the patency of arterial and venous anastomosis in the early phase, the vascular stability of the transferred flap is eventually affected by neovascularization between the donor and recipient tissue. Therefore, vascular development in the contact area is another important factor for flap survival. If the donor tissue has its own vascular supply, this process can be facilitated. Even considering the arterial flow alone, the donor tissue had a vascular component in our cases, and we believe this promoted neovascularization on the contact surface. Furthermore, a harvested donor flap was less limited in size and dimension than a composite graft due to this reliable arterial supply.

Furthermore, toe tissue transfer with the arterial-anastomosisonly technique has some advantages over the conventional anastomosis of both arteries and veins. There is no need for an additional dissection or anastomosis for the veins; thus, simplification of the surgical procedure can be achieved, and the mean operation time would be shortened.

This also helps to reduce the internal damage of a harvested flap. Sometimes a transferred flap requires a smaller and thinner volume of soft tissue for an esthetically pleasing result. As the concerns about supermicrosurgery increase and the techniques continue to be developed, surgical attempts can be made for difficult venous anastomosis in these small flaps. However, dissection for finding venous outflow may cause severe internal injuries. In our method, dissection procedures were simplified to lower the risk of flap damage. Additionally, flaps can have some variations in design because there is no obligation to include veins.

The patient satisfaction results also verified the cosmetically acceptable outcomes. A fingertip is not only functional but also esthetically important for a hand. Because there was no need of additional incisions for the recipient veins, scars were minimized and the patients were satisfied with the results.

There are some disadvantages of arterial-only anastomosis in free toe pulp transfer. Close and frequent observation is needed for early recognition of flap congestion and periodic pin-pricking. It is also difficult to verify the adequate timing and bleeding amount due to the lack of a decongestion guideline. The limitation of this study is related with determining the adequate flap size and volume. We empirically assumed the suitable flap size for artery-only anastomosis, but further studies should be conducted for a general consensus on a maximum flap volume amount to transfer without venous anastomosis.

\section{REFERENCES}

1. Komatsu S, Tamai S. Successful replantation of a completely cut-off thumb: case report. Plast Reconstr Surg 1968;42:374-7.

2. Hattori Y, Doi K, Sakamoto S, et al. Fingertip replantation.J Hand Surg Am 2007;32:548-55.

3. Hattori Y, Doi K, Ikeda K, et al. Significance of venous anastomosis in fingertip replantation. Plast Reconstr Surg 2003; 111:1151-8. 
4. Patradul A, Ngarmukos C, Parkpian V. Distal digital replantations and revascularizations. 237 digits in 192 patients. J Hand Surg Br 1998;23:578-82.

5. Foucher G, Norris RW. Distal and very distal digital replantations. Br J Plast Surg 1992;45:199-203.

6. Akyurek M, Safak T, Kecik A. Fingertip replantation at or distal to the nail base: use of the technique of artery-only anastomosis. Ann Plast Surg 2001;46:605-12.

7. Matsuzaki H, Yoshizu T, Maki Y, et al. Functional and cosmetic results of fingertip replantation: anastomosing only the digital artery. Ann Plast Surg 2004;53:353-9.

8. Li J, Guo Z, Zhu Q et al. Fingertip replantation: determinants of survival. Plast Reconstr Surg 2008; 122:833-9.

9. Bickel KD, Dosanjh A. Fingertip reconstruction. J Hand Surg Am 2008;33:1417-9.

10. Rinker B. Fingertip reconstruction with the laterally based thenar flap: indications and long-term functional results. Hand (N Y) 2006; 1:2-8.

11. Omokawa S, Fujitani R, Dohi Y, et al. Reverse midpalmar island flap transfer for fingertip reconstruction. J Reconstr Microsurg 2009;25:171-9.

12. Wei FC, Colony LH. Microsurgical restoration of distal digital function. Clin Plast Surg 1989; 16:443-55.

13. Buntic RF, Brooks D. Standardized protocol for artery-only fingertip replantation. J Hand Surg Am 2010;35:1491-6.

14. Zhang X, Wen S, Wang B, et al. Reconstruction of circulation in the fingertip without vein repair in zone I replantation. J Hand Surg Am 2008;33:1597-601.

15. Han SK, Chung HS, Kim WK. The timing of neovascularization in fingertip replantation by external bleeding. Plast Reconstr Surg 2002;110:1042-6. 